\title{
Characterization and solvent stable features of strep-tagged purified recombinant lipase from thermostable and solvent tolerant Bacillus sp. strain 42.
}

\begin{abstract}
-A $1.2 \mathrm{~kb}$ lipase gene (AY 78735) from solvent stable and thermostableBacillus sp. strain 42 was overexpressed in a heterologous system that allowed for an extensive characterization of its solvent stability and thermostability. An overexpression was achieved using pET51b vector withEscherichia coli host strain BL21(DE3)pLysS, in which optimum expression was at $22-24 \mathrm{~h}$ incubation at $37^{\circ} \mathrm{C}$, with lipase activity reached at $80.0 \mathrm{U} \mathrm{mL}-1$ (specific activity 160.0 U mg-1), after induction by $0.5 \mathrm{mM}$ IPTG. This expression was 11.5 fold higher and superseded the pQE-30UA/M15 (pREP4) host-vector system, which only achieved at $17.0 \mathrm{U}$ $\mathrm{mL}-1$ (34.0 U mg-1). The fusion lipase contains N-terminal Strep-tag II affinity tag that in one step of purification, the lipase was purified to homogeneity using Strep-tag II agarose column. The lipase was purified at 1.3 fold and $70 \%$ recovery with the elution fraction gave a band of $43 \mathrm{kDa}$ in SDS-PAGE. The purified fusion lipase was most active at $70^{\circ} \mathrm{C}$ and $\mathrm{pH}$ 8.0 , and was stable in a broad $\mathrm{pH}$ range of $7-10$. It showed hydrolysis preference towards olive, sunflower and corn oils. Based on solvent stability studies in 30 min pre-incubation in $25 \% \mathrm{v} / \mathrm{v}$ solvents with a shaking rate at 150 strokes per min, the purified Lip 42 showed a different residual activity profiles depending on solvents and temperatures. Lip 42 was found be stable in polar organic solvents such as DMSO, DMF, acetone, methanol, heptanol and octanol, which could make it as a potential biocatalyst for the use in industrial biodiesel production.
\end{abstract}

Keyword: Solvent stable lipase; Overexpression; Biodiesel; Affinity chromatography; Thermostable lipase. 